\title{
Investigation of Low Density Supersonic Jets by Means of a Molecular Beam Sampling Technique
}

\author{
W. R. Eckelt, H. Hose, and K. SCHÜGERL \\ Institut für Chemische Technologie der Technischen Universität Braunschweig, Germany \\ (Z. Naturforsch. 24 a, 1365-1373 [1969] ; received 6 June 1969)

\begin{abstract}
The performace of a supersonic molecular beam equipment is described. Velocity distributions of the particles in low density supersonic jets consisting of Helium, Argon, Methane and/or Ethane were measured by means of a molecular beam sampling technique and a multi-disc velocity analyser. The jets were produced by expanding the gas from an oven with stagnation pressures of 5 to 5000 torr and temperatures of 293 to $950^{\circ} \mathrm{K}$ across different converging nozzles into a vacuum chamber.

The Mach numbers as functions of the source Knudsen numbers were calculated using the source flow theory of Sherman and the hypersonic approximation for the translational nonequilibrium of Hamel-Willis and Edwards-Cheng. These calculations were carried out for different intermolecular potentials. The values obtained using this theory are compared with the experimental results.
\end{abstract}

Recently there has been a growing interest in molecular beams for aerodynamical, physical and chemical research $1,2,3,4$.

In continuum aerodynamics the collisions among the molecules determines the equation of the state and the transport properties of the fluid. In rarified gases the gas surface collisions also have importance. In the limiting case of free molecule flow, which is the condition of satellite flight, the aerodynamic parameters are completely governed by the surface interaction. Despite its importance in rarified gas dynamics, very little is known about surface interaction. The use of high-energy molecular beams makes it possible to simulate satellite flight by bombarding the surface of the subject with molecular beam particles ${ }^{1,2}$.

In physics, molecular beams are used to experimentally measure the cross sections of atoms and molecules and to estimate the intermolecular potentials $^{3}$.

In chemistry, the measurement of the interaction of the beam molecules with the molecules of the target surface, using molecular beams, makes it

Reprint requests to Prof. Dr. K. Schügerl, Institut für Chemische Technologie der Technischen Universität Braunschweig, D-3300 Braunschweig, Hans-SommerStr. 10.

1 For review article see e.g. J. B. French, Molecular beams for Rariefied Gasdynamic Research, AGARD ograph 113 (1966).

2 For review article see e.g. F. C. Hurlbut, Recent Studies of Particle Surface Interactions, Proc. Atomic and Molecular Beams Conf., Denver 1960. - F. C. Hurlbut, Particle Surface Interaction in Hypersonic Flight, an Annotated Bibliography, Rand Corporation RM-48-85-PR (1966). possible to investigate chemisorption processes and heterogeneous chemical reactions in the most direct way.

By crossing two molecular beams of reaction partners, the elementary steps of complex homogeneous reactions can be investigated ${ }^{4}$. The classical beams have neither the desired intensity nor the necessary energy for most of these investigations. Therefore, new methods were developed to produce molecular beams which were especially suited for these investigations. One of these methods is the formation of molecular beams from supersonic free jets ${ }^{5,6}$.

In the present paper, free jets are used to produce supersonic molecular beams of high Mach number of the particles (i.e. narrow velocity distribution).

The basic principles of producing supersonic molecular beams are well known ${ }^{5,6}$. The most important characteristics of supersonic beam equipment have been presented by several groups? Therefore, this paper disusses only some special characteristics of the equipment used.

3 For review article see e.g. H. Pauly and J. P. Toennies, Advan. Atomic and Mol. Phys. Vol. 1, Academic Press, New York 1965.

4 For review article see e.g. D. R. Herschbach, Advan. Chem. Phys. 10, 319 (1966).

5 A. Kantrowitz and J. Grey, Rev. Sci. Instr. 22, 238 (1951).

6 For review articles see e.g. J. B. Anderson, R. P. Andres, and J. B. Fenn, Molecular Beams ed. (Ross), Interscience Publishers, New York 1966, p. 275. K. Bier, Proc. 3rd Intern. Vacuum Congress, Pergamon Press, London 1966, p. 5.

7 For example K. Bier and O. Hagena, Rarefied Gas Dynamics, Vol. 2 (ed. De Leeuw), Academic Press, New York 1966, p. 260. 


\section{General Considerations}

The gas is expanded through a small nozzle into a vacuum forming a low density supersonic jet with a velocity distribution of particles which is considerably narrower than that of a Maxwellian velocity distribution. During the free jet expansion, the flow undergoes a transition from collision dominated isentropic expansion in the vicinity of the nozzle exit plane to a nearly collision free (free molecular) flow far downstream from the nozzle. The Mach number, along the conter line of the jet, increases according to the collision dominated isentropic expansion until collisions are too infrequent to support the expansion. The equilibrium between the different degrees of freedom cannot be maintained any more. At first, the vibrational, than the rotational and finally the translational temperatures become constant and independent of the distance from the nozzle throut. The Mach number increase now levels off and the expansion is said to "freeze,,.

To describe the complex two dimensional fre jet flow, the process was simplified and approximated by an one dimensional spherical source flow ${ }^{8}$. This model gives a good approximation for high Reynolds numbers but becomes gradually worse during the expansion with decreasing Reynolds numbers.

HAMEL and Willis 9,10 and Edwards and Cheng 11,12 used the hypersonic approximation (i.e. the ratio of the ordered velocity to the thermal velocity is large compared to one) to obtain a consistent truncation of the moment equation in the hypersonic region of the source flow. Thus, they were able to replace the Boltzmann equation by a set of moment equations up to the equation of the stress tensor. The stress tensor is decomposed into a pressure $p_{\|}$along the stream lines and a pressure $p_{\perp}$ perpendicular to the streamlines of the source. Associated with the components of the stress tensor are the kinetic temperatures

$$
T_{\|}=p_{\|} / n k \text { and } T_{\perp}=p_{\perp} / n k
$$

where $n=$ number density of particles and $k=$ Boltzman constant.

8 H. Ashrenas and F. S. Sherman, Rarefied Gas Dynamics, Vol. 2 (ed. De Leeuw), Academic Press, New York 1966 , p. 84.

9 B. B. Hamel and D. R. Willis, Phys. Fluids 9, 829 (1966).

10 D. R. Willis and B. B. Hamel: Rarefied Gas Dynamics, Vol. 1 (ed. Brundin), Academic Press, New York 1967, p. 837.
Thus, the moment equations and their solutions describe the expansion process by two temperatures $T_{\|}$, the temperature along the stream lines, and $T_{\perp}$, the temperature transverse to the stream lines. When $T_{\|} \cong T_{\perp}$ the pressure is isotropic and collision dominated isentropic expansion prevails.

With decreasing frequency of the collision, the expansion becomes increasingly non-isentropic and anisotropic, i.e. the difference between $T_{\|}$and $T_{\perp}$ increases. This theory shows that as the gas expands the perpendicular temperature tends to be cooled by the geometric effect of the expansion and so $T_{\perp}$ decreases more quickly than $T_{\|}$. However, the collisions cause a transfer of energy between the parallel and perpendicular modes of motion. This energy transfer lowers $T_{\|}$and increases $T_{\perp}$. Therefore in the collision dominated region, equipartition exist: $T_{\|} \cong T_{\perp} \sim x^{-4 / 3}$, where $x$ is the distance from the source. With decreasing collision frequency, $T_{\perp}$ still decreases due to geometric expansion, but collisional lowering of $T_{\|}$does not continue and $T_{\|}$ freezes. Downstream from this point the function $T_{\perp} \sim x^{-2}$ would prevail, if absolutely no energy transfer occurs.

However, since $T_{\|} \gg T_{\perp}$, a relatively small collisional transfer of energy from the parallel to the perpendicular direction will have a small effect on $T_{\|}$but a large effect on $T_{\perp}$, hence keeping $T_{\perp}$ on the isentropic curve for great distances and eventually giving rise to asymptotic behaviour $T_{\perp} \sim x^{-1}$. The position $x$ of the transfer from one expansion regime to the other one and the shape of the functions $T_{\|} / T_{0}$ and $T_{\perp}^{\mathrm{V}} / T_{0}$ versus $x / d\left(T_{0}=\right.$ source temperature, $d=$ diameter of the nozzle throat) depends on the assumed intermolecular potential.

The terminal Mach numbers $M_{\|}$and $M_{\perp}$ are given by HAMEL and WILLIS ${ }^{9}$ as functions of the source Knudsen number $K n_{0}=\lambda_{0} / d\left(\lambda_{0}=\right.$ mean free path of the particles in the source) and are

$$
\begin{aligned}
M_{\|} & =\frac{\sqrt{\mathbf{3}\left(1 / K n_{0}\right)^{2 /(3+4 \beta)}}}{\sqrt{y^{0}(\infty)(4.0395)^{3 /(6+8 \beta)}}} \text { and } \\
M_{\perp} & =M_{\|} \frac{\sqrt{x}{\sqrt{2}(1+C)^{(1 / 2 \beta)}}^{\left.\left[y_{0}(\infty)\right]^{(1 / 2} \beta\right)}}{}
\end{aligned}
$$

11 R. H. Edwards and H. K. Cheng, Am. Inst. Aeron. Astronaut. J. 4, 558 (1966).

12 R. H. Edwards and K. H. Cheng, Rarefied Gas Dynamics, Vol. 1 (ed. Brundin), Academic Press, New York 1967, p. 819. 
Here $y_{0}(\infty)$ is a scale variable $\sim 1$ for $T_{\|}$if $x \rightarrow \infty$ and $\beta$ and $C$ are parameters of the intermolecular potential which give the temperature dependence of the modelled collision frequency:

$$
v \cong\left[\frac{T_{\|}+C T_{\perp}}{1+C}\right]^{\beta}
$$

where $C=$ constant

$$
\beta=\frac{1}{2}-2 /(s-1) \text { and }
$$

$s$ is the exponent in the molecular force law: $r^{-s}$

( $r$ is the separation of the particles).

For Maxwell molecules $(s=5) \beta=0$ for pseudo-hard sphere molecules $(s=\infty) \quad \beta=1 / 2$. HAMEL and Willis ${ }^{9}$ gave for these two models the final Mach numbers $M_{\|}(\infty)$ as a function of the inverse Knudsen number for mono-atomic particles $(\gamma=5 / 3)$ :

$\begin{array}{lll}\beta & C & M_{\|}(\infty), \\ 0 & - & 0.421 K n_{0}^{-0.667}, \\ 1 / 2 & 0 & 0.868 K n_{0}^{-0.4}, \\ 1 / 2 & 2 & 0.770 K n_{0}^{-0.4} .\end{array}$

The hard sphere molecules freeze more rapidly and at higher levels than to the Maxwell molecules as illustrated by the following equation:

$$
\frac{T_{\|}(\infty)_{\beta=0}}{T_{\|}(\infty)_{\beta=1 / 2}} \cong\left[\frac{1}{K n_{0}}\right]^{-8 / 15}
$$

The freezing for both $\beta=0$ and $\beta=1 / 2$ is not sudden.

The radial temperature $T_{\perp}$ follows the isentropic expansion $\left(T_{\perp} \sim x^{-4 / 3}\right)$ beyond the point at which $T_{\|}$freezes and then quite abruptly goes to its asymptotic limit $\left(T_{\perp} \sim x^{-1}\right)$.

The small effect of the intermolecular potential on the asymptotic behaviour of $T_{\perp}$ can be seen from the function

$$
\frac{T_{\perp}(\infty)_{\beta=0}}{T_{\perp}(\infty)_{\beta=1 / 2}} \cong\left[\frac{1}{K n_{0}}\right]^{-2 / 15} .
$$

Edwards and Cheng 11,12 using the B-G-K model in the hypersonic limit derived the same results.

ScotT and PhIPps ${ }^{13}$ using a simple near equilibrium assumption estimated the final Mach number $M_{\|}(\infty)$ to be:

$$
M_{\|}(\infty) \sim\left[\frac{1}{K n_{0}}\right] \frac{\gamma-1}{2 \gamma(1-\omega)+2 \omega-1}
$$

13 J. E. Scott, jr. and J. A. Phipps, Rarefied Gas Dynamics, Vol. 2 (ed. Brundin), Academic Press, New York 1967, p. 1337.

14 N. Abuaf, J. B. Anderson, R. P. Andres, J. B. Fenn, and D. R. Miller, Project Squid, Technical Report PR-111-P (1966). where $\mu \sim T^{\omega}$ gives the temperature dependence of the dynamic viscosity $\mu$.

This model gives the same results for the Maxwellian and the hard sphere molecules as the HamelWillis- and/or Edwards-Cheng-model.

Abuaf et al. ${ }^{14}$ and Muntz ${ }^{15}$ extended the theory using the Lennard-Jones $(6-12)$ potential by assuming an ellipsoidal Boltzmann form of distribution function for monoatomic gases. For the Lennard-Jones $(6-12)$-potential, the exponent of the inverse Knudsen number is $0.454^{15}$. Anderson at al. 16,17 found experimentally for Argon particles

$$
M_{\Downarrow}(\infty)=1.17\left(\frac{1}{K n^{0}}\right)^{0.4}
$$

which is in fairly good agreement with the hard sphere model.

Scott and Phipps 13 did not find a constant exponent: for low inverse Knudsen numbers they found a fairly good agreement with the function $M_{\|}(\infty)$ versus $K n_{0}$ for Maxwellian particles and for high inverse Knudsen numbers, they state a fairly good agreement with the hard sphere model. Actually, their data deviate from the latter significantly.

In the present paper experimentally measured Mach numbers are presented as functions of the Knudsen numbers and compared with the theoretical and experimental values of other research groups.

\section{Equipment}

The equipment (Fig. $1 \mathrm{a}$ and b) consisted of a main chamber (H), two beam forming devices (S 1 and S 2) (for crossed beam measurements), an outer tube (1), an inner tube (2), a turntable (3) and a cylindrical baffle (4), which can be cooled by liquid nitrogen or helium.

The cylindrical main chamber (diameter $1000 \mathrm{~mm}$ and height $900 \mathrm{~mm}$ ) and the beam forming tubes (S 1 and S 2) were made of stainless steel $(\mathrm{V} 2 \mathrm{~A})$. The vacuum seal consisted of Viton. The main chamber $(\mathrm{H})$ was evacuated by an oil-diffusion pump (type D 36000, Heraeus) with a pumping capacity of $36000 \mathrm{l} / \mathrm{s}$, which was backed by a mechanical roots pump (type R 1600500 Heraeus) with a pumping capacity of $1600 \mathrm{~m}^{3} / \mathrm{h}$ and a mechanical pump (type DK 180 Heraeus) with a pumping capacity of $180 \mathrm{~m}^{3} / \mathrm{h}$.

15 E. P. Muntz, Rarefied Gas Dynamics, Vol. 2 (ed. Brundin), Academic Press, New York 1967, p. 1257.

16 J. B. Anderson and J. B. Fenn, Phys. Fluids 8, 780 (1965).

17 J.B.Anderson, R.P.Andres, J.B. Fenn, and G. Maise, Rarefied Gas Dynamics, Vol. 2 (ed. De Leeuw), Academic Press, New York 1966, p. 106. 


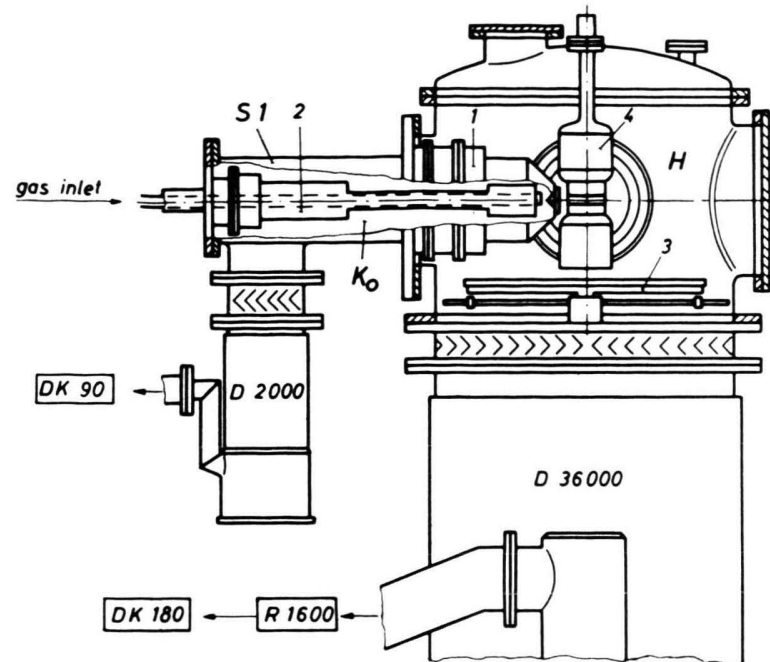

Fig. 1 a. Molecular beam equipment. Side view.

1 outer tube,

2 inner tube,

3 turntable,

4 cylindrical baffle,

D 36000 oil diffusion pump (36000 l/s),

$\mathrm{R} 1600$ roots pump $\left(1600 \mathrm{~m}^{3} / \mathrm{h}\right)$,

DK 180 mechanical pump $\left(180 \mathrm{~m}^{3} / \mathrm{h}\right)$,

D 2000 oil diffusion pump $(2000 \mathrm{l} / \mathrm{s})$,

DK 90 mechanical pump $\left(90 \mathrm{~m}^{3} / \mathrm{h}\right)$.

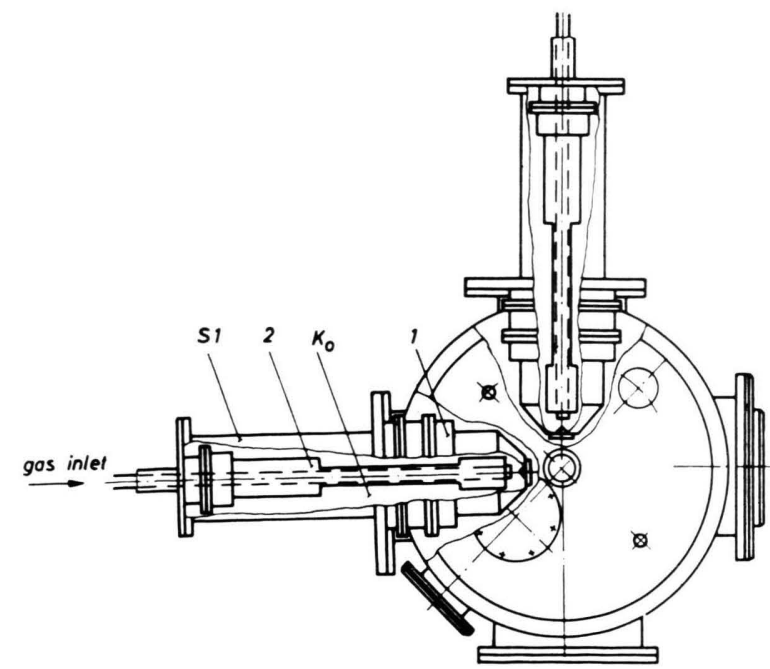

Fig. 1b. Molecular beam equipment. Top view. (For symbols see 1a.)

The main chamber and the oil-diffusion pump were separated by a baffle which was cooled by a two stagerefrigerator (type $\mathrm{KZ} 500 \mathrm{Heraeus}$ ) to about $210^{\circ} \mathrm{K}$. In the main chamber a background pressure of $5 \cdot 10^{-8}$ torr could be achieved. The beam forming devices ( $\mathrm{S} 1$ and S 2) consisted of an outer and an inner tube (Fig. 2). The inner tube was water cooled (4) and had the oven (5) and the nozzle (6) at its free end (Fig. 2). It was connected to a gas inlet

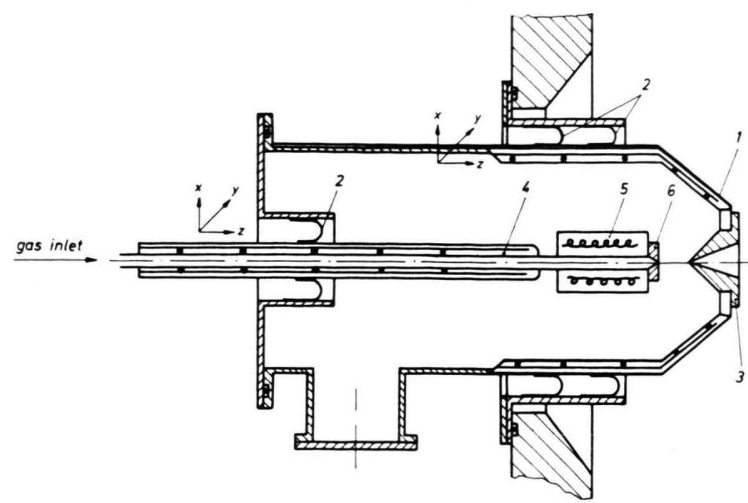

Fig. 2. Beam forming device. 1 water cooled outer tube, 2 flexible Viton collar sealing, 3 skimmer, 4 water cooled inner tube, 5 oven, 6 nozzle.

system. The outer tube, which held the skimmer (3) was also water cooled ( 1 of Fig. 2 ). The inner tube (with the oven and the nozzle) could be moved relative to the outer tube (with the skimmer) in all three directions $(x, y, z)$. The axial distance $(z)$ between nozzle and skimmer could be adjusted between 0.5 and $110 \mathrm{~mm}$ and the radial distances $(x$ and $y$ ) between plus and minus $10 \mathrm{~mm}$ with the accuracy of $0.01 \mathrm{~mm}$.

The position of the whole beam forming device (inner an outer tube) could also be changed relative to the main chamber in all three directions $(x, y, z)$ with the same accuracy.

The nozzle chamber $\left(\mathrm{K}_{0}\right)$ (between the nozzle and the skimmer) was connected to the main chamber only by the skimmer orifice. It was evacuated by an oil-diffusion pump (type DI 2000 Heraeus) with a pumping capacity of $2000 \mathrm{l} / \mathrm{s}$ which was backed by a mechanical pump (type DK 90 Heraeus) with a pumping capacity of $90 \mathrm{~m}^{3} / \mathrm{h}$.

The nozzle chamber was separated from the diffusion pump by a water cooled baffle.

In order to allow adjustment of the beam forming devices (S 1 and $\mathrm{S} 2$ of Fig. 1) relative to the main chamber $(\mathrm{H})$ and of the inner tube relative to the outer tube (Fig. 2), these parts had to be flexibly joined; this sealing was especially difficult. In order to gain this necessary flexibility, a new method has been devised for the sealing. A double Viton collar (2 in Fig. 2) connected the main chamber with the beam forming device and a single Viton collar ( 2 in Fig. 2 ) the inner tube with the outer one.

The nozzle had a throat with a diameter $d$ and a length of $2 d$. The outer and inner angles of the skimmer were constant $\left(50^{\circ}\right.$ and $\left.70^{\circ}\right)$.

The nozzle could be heated up to $750{ }^{\circ} \mathrm{C}$. The constant temperature was maintained by a temperature controller.

\section{Detector, Velocity Selector and the Execution of the Measurements}

A Bayard-Alpert-Detector with an orifice was used for the detection of the molecular beam. A Heraeus ,Ultravac 2 " was used as the power supply. The sensitivity could 
be increased by a factor of ten by decreasing the emission current. The signal was amplified by a DC chopper amplifier and the analoque voltage of the signal was converted into digital values which could printed out. The position of the detector could be changed in two directions $(x, y)$ during the measurements with an accuracy of $0.07 \mathrm{~mm}$. The change of the position was measured by a photocell device.

The molecular beam was chopped at a very low frequency $(0.1$ to $0.01 \mathrm{~Hz})$ and the signals for beam "on" and "off" were separately recorded.

The velocity of the beam particles was measured by a mechanical velocity analyser after Fizeau. The velocity analyser had the following characteristics:

$\begin{array}{lc}\text { number of discs } & 6 \\ \text { diameter of the discs } & 191 \mathrm{~mm} \\ \text { number of slots per disc } & 600 \\ \text { width of the discs } & 1.05 \mathrm{~mm} \\ \text { length of the rotor axis } & 270 \mathrm{~mm} \\ \text { resolving power } & 3.75 \% \\ \text { angular velocity } & 50 \text { to } 450 \mathrm{rps} \text {. }\end{array}$

The angular velocity of the analyser was also measured by a photocell device. The number of pulses were counted and printed out parallel with the beam signal (Fig. 3).

The velocity analyser and the detector were mounted on the turn table (Fig. 4). By rotating the table one could use the velocity analyser-detector-system for the analysis of both of the beams which cross each other at the angle of $90^{\circ}$.

The velocity analyser could be moved into the beam path during the measurements by means of a flexible metal device of Bellow Co.

To measure the spatial distribution of the beam particles, the detector was moved perpendicular to the beam axis $(x$ or $y)$ and the intensity of the beam was recorded at different distances from the nozzle.

For the measurement of the velocity distributions, the angular velocity of the velocity analyser was increased until no partieles could pass it. The power supply was then switched off and the angular velocity of the analyser

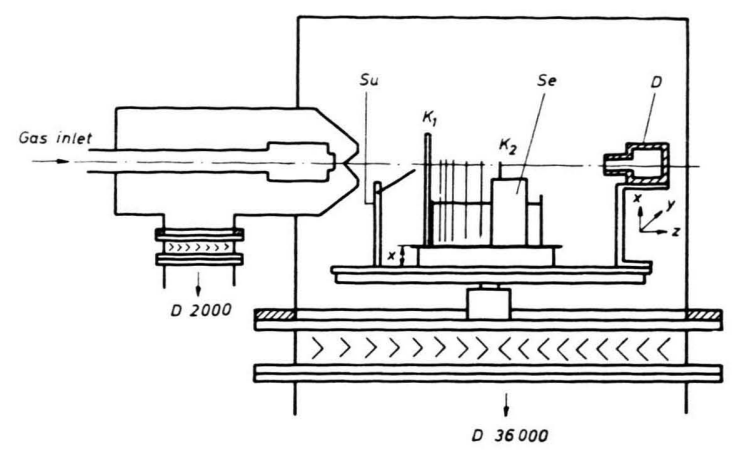

Fig. 4. Setup for the measurements of the velocity distribution of beam particles. $\mathrm{D}$ detector, $\mathrm{K}_{1}, \mathrm{~K}_{2}$ collimators, Se velocity selector, Su beam chopper, $x \uparrow$ indicates that the velocity selector can be raised into the beam path during the measurement.

slowly diminished. The angular velocity of the analyser and the beam signal were recorded simultaneously. During these measurements the molecular beam was chopped.

\section{Operation Characteristics}

The operating conditions for the beam formation were:

$$
\begin{array}{ll}
\text { nozzle diameters } d: & 250,90 \text { and } 50 \text { micron, } \\
\text { skimmer diameter: } & \mathbf{7 5 0} \text { and } 500 \text { micron }
\end{array}
$$

The temperature $T_{0}$ of the oven-nozzle system was varied between 20 and $700{ }^{\circ} \mathrm{C}$, stagnation pressure $p_{0}$ between 5 and 5320 torr, background pressure $p_{1}$ in the nozzle chamber, between $10^{-3}$ and $10^{-6}$ torr and background pressure $p_{2}$, in the main chamber, between $10^{-6}$ and $5 \cdot 10^{-8}$ torr.

The distance were:

$$
\text { nozzle-skimmer: variable }
$$

skimmer-collimator 1: $\quad 102.3 \mathrm{~mm}$

skimmer-collimator 2: $\quad 220.5 \mathrm{~mm}$

skimmer-detector: $\quad 340.5 \mathrm{~mm}$.

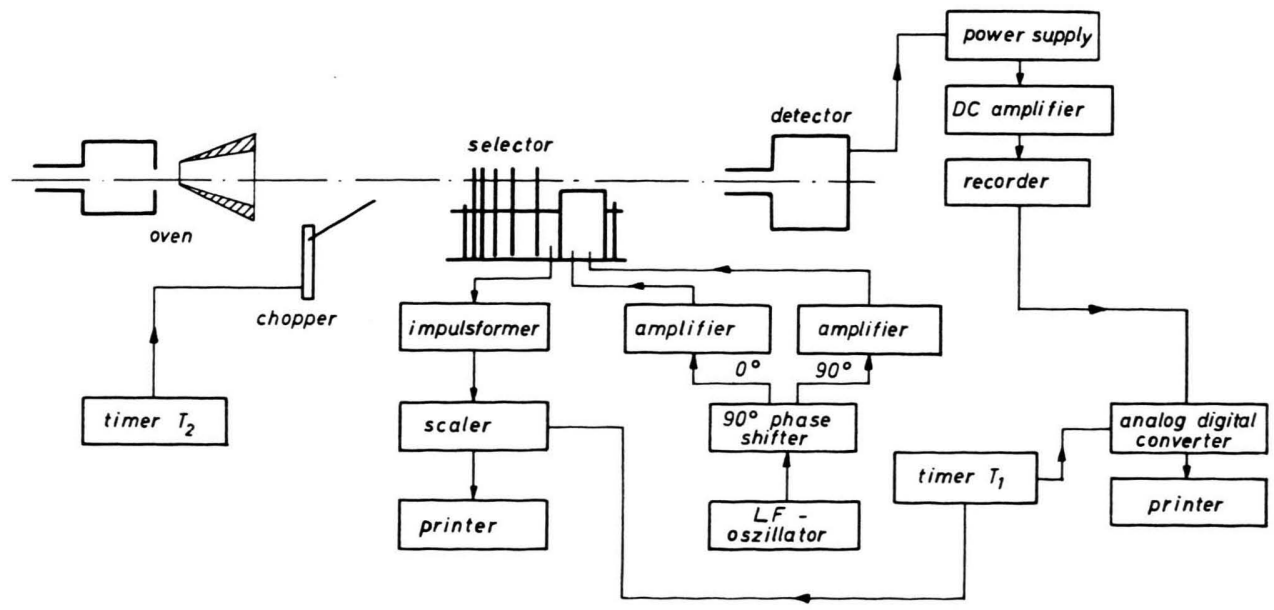

Fig. 3. Schematic flowsheet for the measurement of spatial and velocity distribution of beam particles 


\section{Intensity of the Beam}

Fig. 5 and 6 shows typical spatial distributions of beam intensities for different nozzle-skimmer distances, which were measured with different resolving powers of the detector.

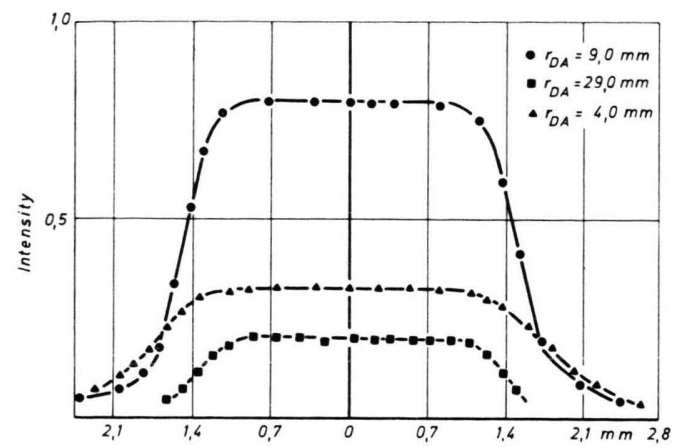

Fig. 5. Typical beam profiles I; diameter of detector opening $0.3 \mathrm{~mm}, p_{0}=5200$ Torr, $T_{0}=750^{\circ} \mathrm{K}$, diameter of collimator opening $1.2 \mathrm{~mm}$, distance between skimmer and detector $250 \mathrm{~mm}$.

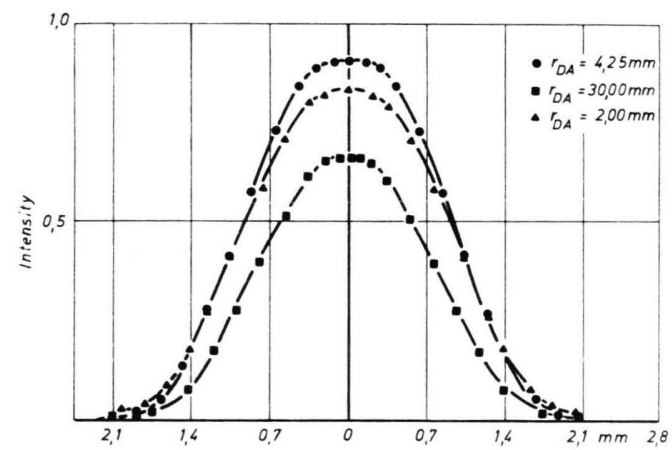

Fig. 6. Typical beam profiles II; diameter of detector opening $1.5 \mathrm{~mm}, p_{0}=100$ Torr, $T_{0}=950^{\circ} \mathrm{K}$, diameter of collimator opening $0.6 \mathrm{~mm}$, distance between skimmer and detector $250 \mathrm{~mm}$.

If the diameter of the orifice is small (e.g. $0.3 \mathrm{~mm}$ ), one finds the expected trapezoid distribution of the beam intensity (Fig. 5). With a large orifice (e.g. $1.5 \mathrm{~mm}$ ), the intensity distribution follows approximately that of an error function (Fig. 6).

The distance between the nozzle and the skimmer was varied for a given stagnation pressure $p_{0}$ and oven temperature $T_{0}$ and the optimal distance $r_{\text {opt }}$ was estimated for the highest intensity of the molecular beam.

Figure 7 shows typical intensity $I$ versus nozzleskimmer distance $r_{\mathrm{DA}}$ functions for different stagnation pressures $p_{0}$. One can recognize the strong dependence of the intensity on $r_{\mathrm{DA}}$. In the neighbourhood of $r_{\text {opt }}$ the intensity changes only slightly

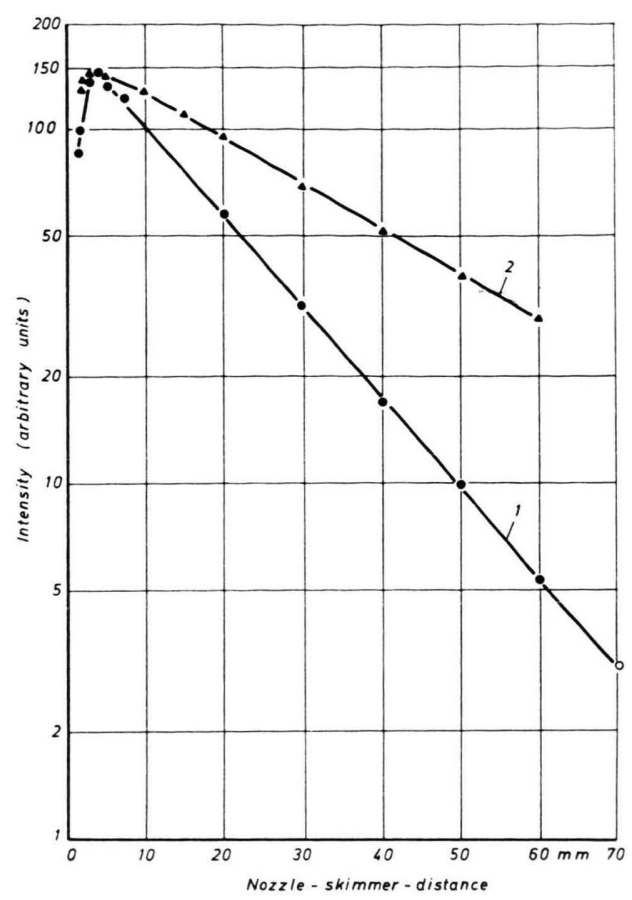

Fig. 7. Example for beam intensity as function of nozzleskimmer-distance. $\mathrm{N}_{2}$-beam. Distance between skimmercollimator $102 \mathrm{~mm}$, distance between skimmer-detector $244 \mathrm{~mm}$, nozzle diameter $250 \mu$, nozzle temperature $800^{\circ} \mathrm{K}$, diameter of collimator opeing $1000 \mu$, diameter of skimmer opening $500 \mu$.

$$
\begin{aligned}
& \text { 1: } p_{0}=107 \text { Torr, } p_{1}=4.5 \cdot 10^{-4} \text { Torr; } \\
& \text { 2: } p_{0}=27 \text { Torr, } p_{1}=1.8 \cdot 10^{-4} \text { Torr. }
\end{aligned}
$$

with increasing $r_{\mathrm{DA}}$. The influence of the stagnation pressure $p_{0}$ on the intensity $I$ depends on the background pressure $p_{1}$ in the nozzle chamber.

If $p_{1}$ is high, $I$ decreases with increasing $p_{0}$, since $p_{1}$ also increases and $I$ is an exponential function of $p_{1}$. For low $p_{1}\left(<10^{-3}\right.$ torr $), I$ increases with increasing $p_{0}$ (Fig. 8).

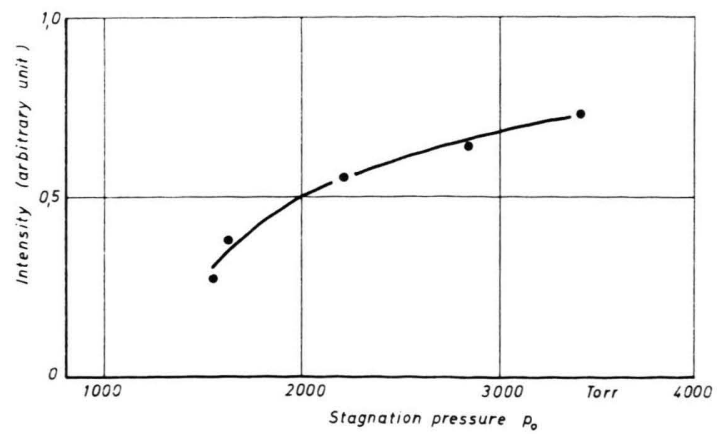

Fig. 8. Example for the beam intensity as function of the stagnation pressure $p_{0}$ measured at optimal nozzle-skimmer distance. $\mathrm{C}_{2} \mathrm{H}_{6}$-beam. Nozzle temperature $500{ }^{\circ} \mathrm{K}$, nozzle diameter $50 \mu$, diameter of skimmer opening $750 \mu$. diameter of collimator opeing $600 \mu, p_{1}<1.5 \cdot 10^{-3}$ Torr. 
In order to estimate the absolute intensities, the detector was calibrated with classical molecular beams. The following typical intensities were measured with a detector-skimmer distance of $340.5 \mathrm{~mm}$.

\begin{tabular}{lc}
\hline \multicolumn{1}{c}{ Gas } & molecules/sec sr. \\
\hline Helium & $2 \times 10^{17}$ \\
Methane & $1 \times 10^{17}$ \\
Ethane & $7.9 \times 10^{16}$ \\
Argon & $6.9 \times 10^{16}$ \\
\hline
\end{tabular}

\section{Velocity Distribution of the Beam Particles}

Because of the insufficient resolving power $(3.75 \%)$ of the velocity analyser, the measured velocity distributions had to be corrected. This correction was carried out by numerical integration of the theoretical velocity distribution for the range of the unresolved velocities. The error, caused by the insufficient resolving power, in the measured relative half width ( $\mathrm{rhw}$ ) of the velocity distribution is $9 \%$ for rhw equal to 8.8 .

Figure 9 shows some measured most probable velocities, which are equal to the mean velocities for these high stagnation pressures, for Argon, Ethane,

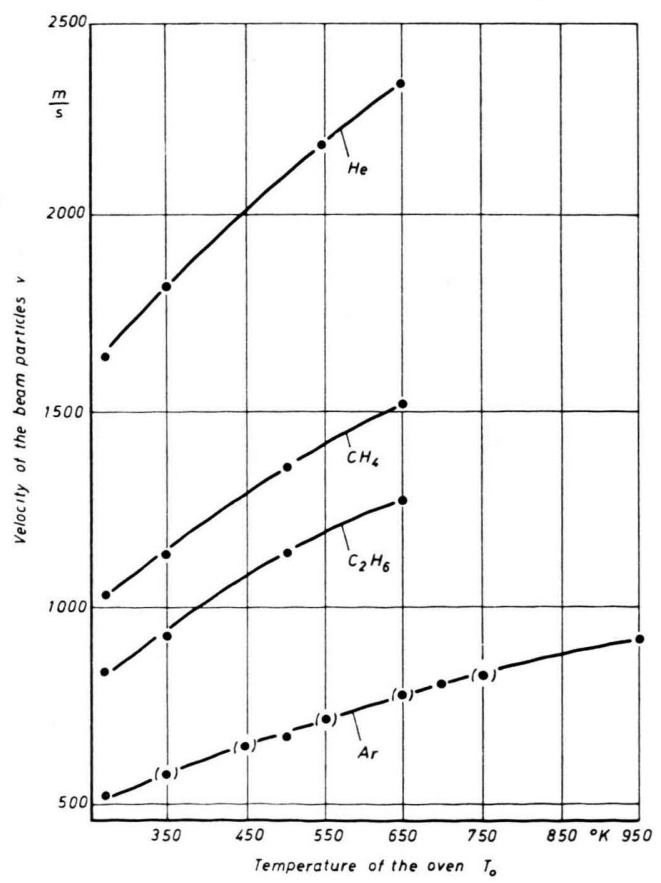

Fig. 9. Example for the mean (and for most probable) velocity of beam particles as function of the oven-temperature for high $p_{0}$. Nozzle diameter $50 \mu$, diameter of skimmer opening $750 \mu$.
Methane and Helium versus, temperature $T_{0}$ of the oven.

These velocities depend only slightly on the pressure ratio $p_{0} / p_{1}$. The half width of the velocity distribution decreases with incrrasing pressure ratio $p_{0} / p_{1}$ for constant temperature $T_{0}$ (Fig. 10) and increases at constant pressure ratio $p_{0} / p_{1}$ with an increase in temperature $T_{0}$ (Fig. 11).

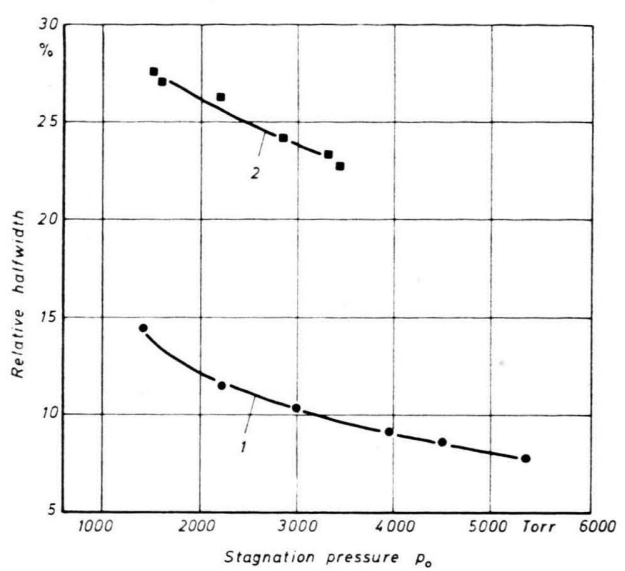

Fig. 10. Example for the relative half width of velocity distribution as function of the stagnation pressure $p_{0}$. 1 : Argon beam, oven temperature $750^{\circ} \mathrm{K} ; 2$ : Ethane beam, oven temperature $500^{\circ} \mathrm{K}$.

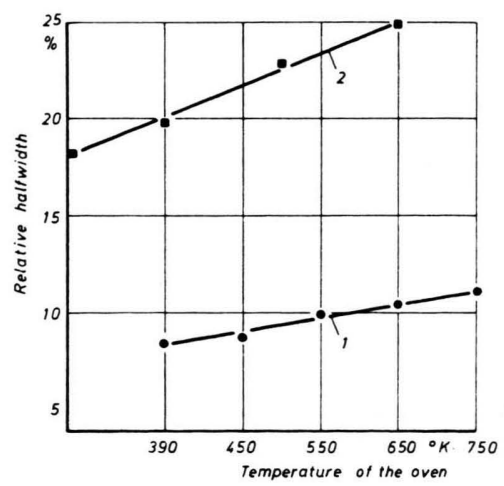

Fig. 11. Exemple for the relative half width of the valocity distribution as function of the oven temperature. 1: Argon beam, $p_{0}=1520$ Torr; 2 : Methane beam, $p_{0}=1620$ Torr.

It is possible to keep the relative width of the velocity distribution for every oven temperature (i.e. every mean velocity) constant, if one appropriately increases the presure ratio $p_{0} / p_{1}$ with $T_{0}$.

\section{Discussion of Results}

From the measured velocity distribution functions, the parallel Mach numbers $M_{\|}\left(\left(M_{\|}^{2}=\right.\right.$ 
$u^{2} m / \gamma k T_{\|}, u=$ mean flow velocity of particles, $\gamma=c_{p} / c_{v}, c_{p}$ and $/$ or $c_{v}=$ specific heat for constant pressure and/or volume, $m=$ mass of the particles) and the parallel temperatures $T_{\|}$were estimated ${ }^{18}$.

Figure 12 shows some of the results for Argon with different Knudsen numbers in the form of the ratio $T_{\|} / T_{0}$ as a function of the dimensionless distance $x / d$ from the nozzle. One can recognize that the parallel temperature $T_{\|}$does not freeze suddenly with increasing $x$. The curve 1 (for $K n_{0} \cong 6 \cdot 10^{-3}$ ) fits fairly well with the theoretical curve with $\beta=0.35$ and $C=0$. The curve 2 (for $K n_{0} \cong 2.5 \cdot 10^{-3}$ ) is not consistent with the theoretical curve with the same $\beta$. For this Knudsen number, a much better agreement between the theoretical and experimental curves can be found for a $\beta$ which is much grater than 0.5 .

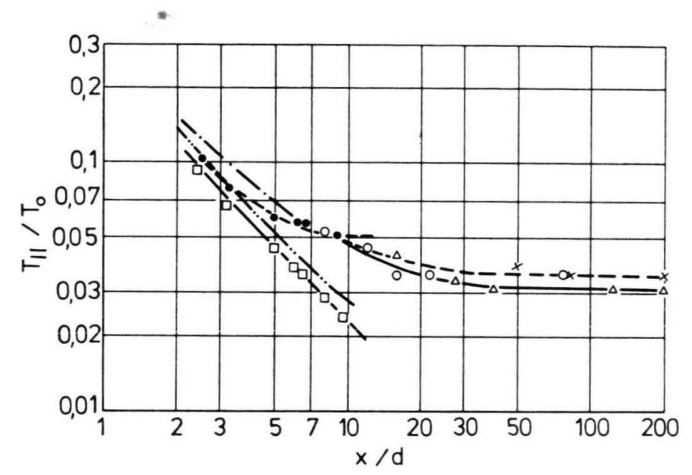

Fig. 12. Dimensionless parallel temperature $T_{\|} / T_{0}$ of the particles as function of the dimensionless distance from the nozzle $x / d$ for Argon and Helium.

Argon:

Symbol

$p_{0}$ (torr) $d(\mu)$

$T_{0}\left({ }^{\circ} \mathrm{K}\right)$

$K n_{0}$

$R e_{0}$

Helium $^{15}: \bullet K n_{0}=5.3 \cdot 10^{-3}, \square K n_{0}=2,0 \cdot 10^{-3}$.

- . - - - theoretical curve for $\beta=0.353$,

$-\cdot-\cdot$ - theoretical curve for $\beta=0.353$,

$$
K n_{0}=5.3 \cdot 10^{-3} ;
$$

- $\cdots-\cdots$ - theoretical curve for $\beta=0.353$,

$$
K n_{0}=2 \cdot 10^{-3} \text {. }
$$

Figures 13 and 14 show the final parallel Mach numbers $M_{\|}(\infty)$ as functions of the inverse source Knudsen numbers $K n_{0}{ }^{-1}$ for Helium and Argon.

Figures 15 and 16 show the same functions for Methane and Ethane. These functions can be described by a simple power function such as

$$
M_{\|}(\infty)=B \cdot K n_{0}^{-\alpha}
$$

where $B$ and $\alpha$ are constants.

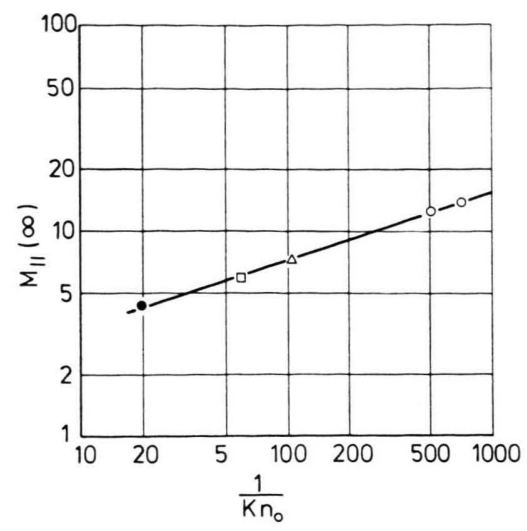

Fig. 13. Final parallel Mach number as function of the inverse Knudsen number for Helium.
Symbol $T_{0}\left({ }^{\circ} \mathrm{K}\right)$
$d(\mu)$

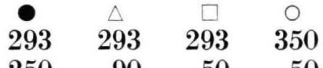

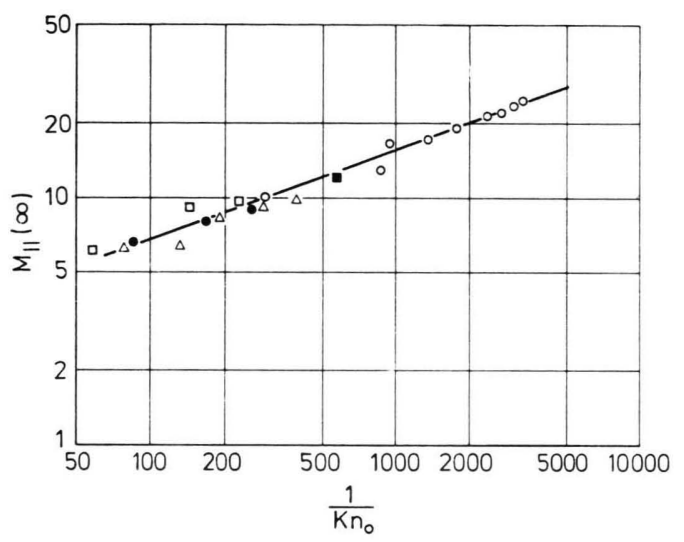

Fig. 14. Final parallel Mach number as function of thə inverse Knudsen number for Argon

$\begin{array}{lccccc}\text { Symbol } & \bigcirc & \bullet & \triangle & \square & \mathbf{\square} \\ T_{0}\left({ }^{\circ} \mathrm{K}\right) & 750 & 750 & 950 & 700 & 700 \\ d(\mu) & 50 & 250 & 250 & 50 & 90\end{array}$

From the Figs. 13 through 16, the following exponents can be estimated:

$$
\begin{aligned}
& \mathrm{He}: \alpha=0.33, \quad \mathrm{Ar}: 0.35, \\
& \mathrm{CH}_{4}: 0.22 \text {, and } \mathrm{C}_{2} \mathrm{H}_{6}: 0.21 .
\end{aligned}
$$

For $\mathrm{He}$ and $\mathrm{Ar}$, which have only translational energy, these exponents were calculated using different potential models. To get the exponents experimentally found, one has to set (with $C=0$ ) $\beta=0.67$ for Argon and $\beta=0.75$ for Helium. This $\beta$ for Argon ist not consistent with the one evaluated from fitting the theoretical function $T_{\|} / T_{0}$ versus

18 E. L. Knuth and S. S. Fisher, J. Chem. Phys. 48, 1674 (1967). 


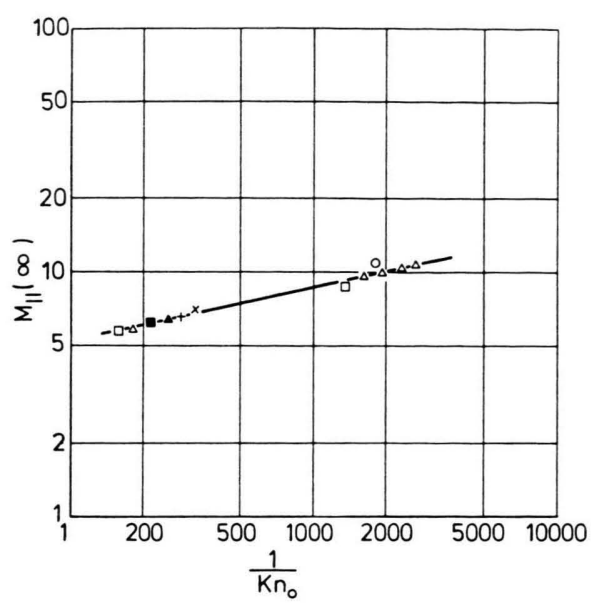

Fig. 15. Final parallel Mach number as function of the inverse Knudsen number for Methane.

$\begin{array}{ccccccccc}\text { Symbol } & \circ & \triangle & \square & \bullet & \mathbf{\Delta} & \mathbf{b} & \times & + \\ T_{0}\left({ }^{\circ} \mathrm{K}\right) & \mathbf{3 5 0} & \mathbf{5 0 0} & \mathbf{6 5 0} & \mathbf{3 5 0} & \mathbf{5 0 0} & \mathbf{6 5 0} & \mathbf{5 0 0} & \mathbf{6 5 0}\end{array}$

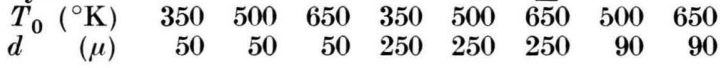

$x / d$ with the experimental one for high Knudsen numbers. With decreasing Knudsen number (increasing Reynolds number $\left.R e_{0}=\left(\mu_{0} d / \nu_{0}\right)\right)$ the agreebetween the two $\beta$ 's improves.

Because only very few functions $T_{\|} / T_{0}$ versus $x / d$ have been published, and these have been measured under different conditions, it is difficult to compare the results of the different groups.

The results of this paper do not seem to be consistent with those of Anderson et al. 16,17, Abuaf et al. ${ }^{14}$ and Muntz ${ }^{15}$ for great distances from the nozzle.

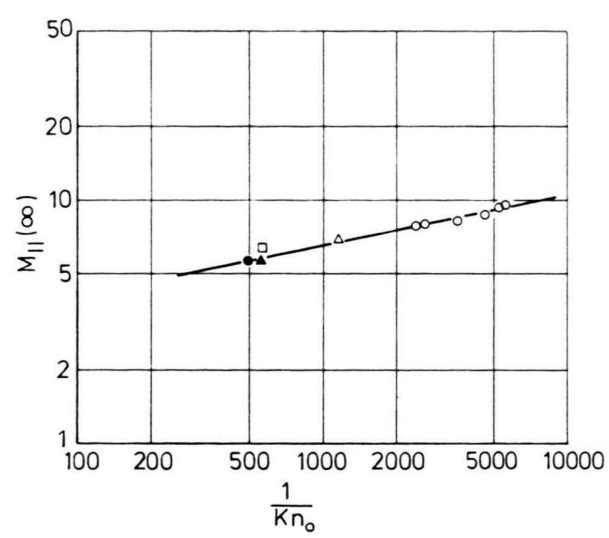

Fig. 16. Final parallel Mach number as function of the inverse Knudsen number for Ethane.

$$
\begin{aligned}
& \begin{array}{lccccc}
\text { Symbol } & \square & \bullet & \bigcirc & \Delta & \Delta \\
T_{0}\left({ }^{\circ} \mathrm{K}\right) & 500 & 500 & 500 & 650 & 650
\end{array} \\
& \begin{array}{llrrrrr}
d^{0} & (\mu) & 90 & 250 & 50 & 250 & 90
\end{array}
\end{aligned}
$$

The exponent $\alpha$ for Argon evaluated from the function $M_{\|}(\infty)$ versus $\left(1 / K n_{0}\right)$ in this paper is smaller than the one found by ANDERSon and/or ABUAF $(\alpha=0.4)$ and consistent with the data of ScotT and Phipps ${ }^{13}$ for high inverse Knudsen numbers.

For Methane and Ethane which also have internal degrees of freedom no other experimental results exist and there is no theory to compare our result with.

Acknowledgement. The authors wish to acknowledge their gratitude to the Deutsche Forschungsgemeinschaft and to the Stiftung Volkswagenwerk for financial support. 